\title{
Contrasts and similarities between two audio research communities in evaluating auditory artefacts
}

\author{
Mariana Seiça \\ CISUC, Department of Informatics Engineering \\ Coimbra, Portugal \\ marianac@dei.uc.pt \\ Pedro Martins \\ CISUC, Department of Informatics Engineering \\ Coimbra, Portugal \\ pjmm@dei.uc.pt
}

\author{
Licínio Roque \\ CISUC, Department of Informatics Engineering \\ Coimbra, Portugal \\ lir@dei.uc.pt \\ F. Amílcar Cardoso \\ CISUC, Department of Informatics Engineering \\ Coimbra, Portugal \\ amilcar@dei.uc.pt
}

\begin{abstract}
The design of auditory artefacts has been establishing its practice as a scientific area for more than 20 years, with a crucial element in this process being how to properly evaluate acoustic outputs. In this paper, we sought to map the evaluation methods applied in a general search inside two main audio-focused conferences: Audio Mostly and the International Conference on Auditory Display (ICAD). Revisiting last year's editions, as well as a keyword-based search in the last ten years, we attempted to gather and classify each evaluation method according to the level of user involvement, their role, and the authors intentions in using each method. We propose an initial mapping for this gathering, in a framework of evaluation approaches which can reinforce and expand current practices in the creation of auditory artefacts.
\end{abstract}

\section{CCS CONCEPTS}

- Human-centered computing $\rightarrow$ Auditory feedback; HCI design and evaluation methods.

\section{KEYWORDS}

evaluation method, literature review, interacting with audio, auditory display

\section{ACM Reference Format:}

Mariana Seiça, Licínio Roque, Pedro Martins, and F. Amílcar Cardoso. 2020. Contrasts and similarities between two audio research communities in evaluating auditory artefacts. In Proceedings of the 15th International Audio Mostly Conference (AM'20), September 15-17, 2020, Graz, Austria. ACM, New York, NY, USA, 8 pages. https://doi.org/10.1145/3411109.3411146

\section{INTRODUCTION}

The Audio Mostly Conference appeared for the first time in 2006, in Piteå, Sweden, with 15 editions of interdisciplinary work in sound design, interaction and experience with sound. Exploring new theories and experience on sound design practices, the community

\section{(c) (7) (2)}

This work is licensed under a Creative Commons Attribution-ShareAlike International 4.0 License. AM'20, September 15-17, 2020, Graz, Austria

(c) 2020 Copyright held by the owner/author(s).

ACM ISBN 978-1-4503-7563-4/20/09.

https://doi.org/10.1145/3411109.3411146 seeks to embrace techniques for sonic interaction and the conception of strong sonic elements in interface design, with its findings published in the ACM Digital Library.

The International Community of Auditory Display (ICAD), on the other hand, has an older legacy, with its first edition tracing back to 1992, where the study of auditory displays was proposed as a scientific field. The focus of the community was to explore the "use of non-speech audio to represent information" [29], refining and developing sonification techniques, with applications and knowledge from multiple areas that form its interdisciplinary nature. However, "despite some innovative one-off successes, the widespread adoption of sonification to present complex data has largely failed to materialize" [47]. Proper evaluation of auditory artefacts is one key issue of discussion and reflection as a crucial medium to bring validation and consistency to auditory display as a research field, and not just "entertaining curiosity" [47].

Last year, one of the authors of the Sonification Report (the key report published in 1999 to identify the field's focus and research agenda [35]), reflected upon the lingering challenges auditory displays face, and possible shifts for evolution and establishment of the field [47]. Neuhoff highlighted several continuing challenges, from (1) the difference between vision and audition in terms of precision and (2) between each listener with his/her individual characteristics, (3) the interaction between each auditory dimension and how they influence each other, (4) the musical background of many researchers which may restrain sonification designs, (5) and the intrinsic interdisciplinary nature of sonifications. This last issue particularly brings out the evaluation stage, questioning how can it be devised: by the designer alone, through participatory design processes, or by statistical analysis of listening preferences and task success [47]. With the current work, we intend to take stock of the diversity of approaches across communities by comparing two practices, possibly motivated also by diverse community views on what should be their object of study, possible design objects and their relative value, the subjects involved in experiencing and assigning value, meaning or fitness for purpose, the evaluation models and references influencing their approach, etc. 


\subsection{Evaluating auditory artefacts and beyond}

In 2008, Hermann's definition of sonification provided a more solid, aggregating description of the underlying drawbacks, peculiarities and tendencies of the field, formulated as being reflective of "objective properties of the input data (...), have a systematic transformation method (...) and be reproducible" [28]. Yet, the quest for "sample-based identity" through a defined, repeatable structure may turn out to be restrictive in interpretive, exploratory opportunities. Does it weaken the communicational, functional purpose to devise a flexible sound structure? This characteristic was already questioned as a constraint in musical mapping [65], giving an example of the Listening to the Mind Listening at ICAD $2004^{1}$. Several proposed pieces resembled electroacoustic compositions, which leads us to the recurrent debate on the limits between sonification and music [65]. One argument against it defends that the transformation of a sonification into a musical composition can disconnect the attentive attitude needed for the listener to interpret the underlying data. However, the search for musical forms in the data patterns can provide an aesthetic quality that engages the listener and awakens an active listening attitude of truly listening. "Is sonification art, design, science, or a mixture of all three?" - Neuhoff's question encompasses these reflections, departing from sonification as an accurate, reproducible scientific method to embrace its relationships with art and design. In line with this union, evaluating auditory artefacts must expand its pure statistical and technical analysis, author-only focus and data-to-mapping accuracy to adopt new general methods of designing and evaluating the listener's experience.

The focus on the design of the listener's experience has been a goal more often adopted in the Audio Mostly community, with explorations and creation of audio-based interfaces exploring broader acoustic concepts of audio storytelling, musical instruments, soundscape composition and sonic interaction.

With these two knowledge-seeking paths in mind, our first intention is to paint a general picture of the evaluation methods applied in each community, distinguishing and contrasting different perspectives and possible trends within both communities. Our second goal is to foresee how can these be combined in a methodological framework for evaluation, considering expected results and forms of user involvement, while bridging the interdisciplinary definition of both communities through the evaluation methods.

The remainder of this paper is structured as follows. Section 2 comprises an overview of the literature review and the thematic analysis undertaken to assess the evaluation methods. Section 3 presents the proposed framework for evaluation possibilities. Section 4 addresses the potential of this proposal, reflecting on its implications and connections with each evaluation method. The fifth and final section concludes the paper, listing the findings and future directions to take.

\section{LITERATURE REVIEW}

We conducted a contrasting review of Audio Mostly and ICAD state-of-the-art regarding the full paper submissions of the latest editions, namely the proceedings of the 14th International Audio Mostly Conference: A Journey in Sound, and the proceedings of

${ }^{1}$ Listening to the Mind Listening - https://www.icad.org/websiteV2.0/Conferences/ ICAD2004/concert.htm the 25th International Conference on Auditory Display, which both took place in 2019. This gathering resulted in 29 papers from Audio Mostly and 38 from ICAD.

In addition, we performed a general search of full papers published in the last ten years in both conferences, filtered by having the words "evaluation" or "evaluating" in the title and/or abstracts. We found 10 papers in Audio Mostly and 16 in ICAD, gathering a total of 39 papers from Audio Mostly and 54 from ICAD.

In total, 93 papers were reviewed for this proposal. From the 93 results, 30 did not include any kind of evaluation method, which resulted in 63 remaining papers for analysis, 26 from Audio Mostly and 37 from ICAD.

The criteria employed in the literature search was thereby based on these two sets: a single edition of full papers, with the goal of gathering a complete evaluation portrait of a recent edition, plus an opportunistic keyword-based and evaluation-related search to retrieve specific references, which would complement possible tendencies taken from the history of each conference. It should be noted that this was a first iteration of this literature review, aimed at providing a generalized first portrait of both conferences, from which we could draw a map of possibilities and attempt to categorize different methods.

\subsection{Thematic analysis: evaluation methods}

All the papers were reviewed to list:

(1) the evaluation method(s) applied in each study;

(2) the authors' intention for choosing the method(s);

(3) the type of results found in each case.

Focusing on the methods employed, we counted the number of times each method appeared in a paper, as properly defined and applied by the authors. The main keywords for each method and respective counts are listed in Table 1, specifying in which conference the respective paper was published.

The first method considered were listening tests, which consist of reproducing the result while subjecting it to a quality recognition or distinction test by passive listeners. Analysing the resulting table, around $65 \%$ of ICAD's reviewed papers $[1,2,4,6,8,11,20$, $22,25-27,33,34,36,40,42,43,45,50,52,57,60,68,72]$ use this method, which shows a clear tendency in ICAD's evaluation choices compared to only $23 \%$ of listening studies used in Audio Mostly $[9,10,16,41,51,71]$.

User studies incorporate more complex procedures from the user than just passive listening through an embodied, active participation. This method has a more balanced number of applications between communities, with $27 \%$ of papers in Audio Mostly describing user tests $[21,23,24,48,49,58,59]$ and $24 \%$ in ICAD $[7,19,27,36,38,46,56,62,69]$. A specific type of user test is implemented in a game-based experience, drawing conclusions from the player's experience through playtesting. Five examples applying this method were found, two in Audio Mostly $[49,58]$ and three in ICAD [7, 27, 36].

Nine examples were found whose evaluation was focused on algorithmic or system performance, without a user-based evaluation, retrieving quantitative results of a system performing with different sets of input parameters, and/or on different contexts. Only 
Table 1: Compilation of the resulting count of methods

\begin{tabular}{|c|c|c|c|}
\hline KEYWORDS & RESULTS & $\begin{array}{l}\text { Audio } \\
\text { Mostly }\end{array}$ & ICAD \\
\hline listening & 30 & 6 & 24 \\
\hline user & 16 & 7 & 9 \\
\hline algorithmic performance & 9 & 6 & 3 \\
\hline interview & 6 & 3 & 3 \\
\hline survey & 5 & 2 & 3 \\
\hline workshop & 4 & 3 & 1 \\
\hline playtesting & 5 & 2 & 3 \\
\hline questionnaire & 3 & 1 & 2 \\
\hline content analysis & 3 & 1 & 2 \\
\hline observation & 2 & 2 & - \\
\hline $\log$ files & 2 & 1 & 1 \\
\hline performance & 2 & 2 & - \\
\hline grounded theory & 1 & - & 1 \\
\hline phenomenological & 1 & - & 1 \\
\hline video & 1 & 1 & - \\
\hline ethnographic & 1 & 1 & - \\
\hline case study & 1 & 1 & - \\
\hline
\end{tabular}

$8 \%$ included this evaluation method in ICAD [30, 66, 70], compared to $23 \%$ in Audio Mostly [13, 15, 18, 37, 44, 73].

Online or in-person surveys are generally comprised of closedended questions, using scales (as the Likert scale, for example) for rating a given characteristic or statement about the experience. A balanced number of examples using this method was found in both conferences, with two in Audio Mostly [54, 58] and three in ICAD $[4,34,56]$. The similar term questionnaire was also mentioned in three examples $[1,43,51]$.

\subsection{Alternative Qualitative Approaches}

A few examples of qualitative data collection methods were also found, focused on spoken or written reports and audio-visual records of the experience.

The interview method, usually composed of closed- and openended questions of "why" or "how", is used to retrieve the user's conscious opinion of a certain experience, with the majority of question usually predefined to gain insights about specific goals. Six examples were found, three in each conference [5, 6, 38, 49, 59, 74].

Exploratory observation can also be included in the evaluation to "draw context-specific features"[12] and observe the user's behavior during the experimentation, with two papers of Audio Mostly reporting its use [5, 49].

Video analysis is used to detain a complete record of the user's experience, for deeper analysis of interactions and reactions which might be missed during observation. One single example was found in Audio Mostly [5].

The use of participatory design workshops brings the user to the design process, either field experts with different knowledge perspectives or people from the general audience, providing opportunities for creative collaborations and an environment for experimentation and discussion. By involving the end-user in the creation side of design, participatory design engages their unique perspectives in suggesting "alternative interpretations of what's really going on or what should really be going on around a system, other than the designer's intention for it" [61]. This method was found in three examples in Audio Mostly [3, 31, 75], which corresponds to $12 \%$ of the papers, and just one in ICAD [67].

The performance method is mainly used for devising new instruments for musical expression, which requires not only demonstrations of musical scores written for them, but also performances using them to ensure their functionality in a practical scenario as proofs of concept. Two examples of this application were found in Audio Mostly [31, 64]. One possible reason for this scarce number might be that this specific goal of instrument creation is not a main goal of research in these two communities. However, we know this to be a common evaluation requirement in the New Instruments for Musical Expression (NIME) community, as shown by two examples found in last year's edition $[17,53]$.

\subsection{Methods for data analysis}

The form of analysis of the data collected from each method can also be done in several ways, either quantitatively, or qualitatively. The listening tests through goal-oriented task require statistical analysis of success rates. Log files are a form of saving technical information about a user interaction, as the date, time or patterns/movements of an interaction session. Two examples were found which applied usage logs, one in Audio Mostly [49] and one in ICAD [55].

Entering the spectrum of open-ended questions and interviews require a qualitative analysis through content and emerging themes. Two studies in ICAD adopted this kind of content analysis [6, 60], and just one in Audio Mostly [75].

Other methods were found that fall into more generic qualitative approaches, namely grounded theory [14], collecting themes, sensations, actions and the user's behavior to form a general theory about the artefact; phenomenology, intended to analyse the experience as a phenomenon and how the users perceive it [32, 39]; case studies, which allows the study of a certain phenomenon in a real-life context, gathering the perspectives of everyone involved; and ethnographic research, integrating a certain community for a limited time to study their practices. Four examples were found, one for each method, the first two in $\operatorname{ICAD}[25,60]$ and the other two in Audio Mostly [5, 63].

Though this review can be considered an insufficient sample to withdraw major conclusions, a clear past tendency can be seen in the ICAD community to implement quantitative measures through passive listening experiments for their testing purposes. We are not 
arguing that a quantifiable measure of experience and mapping success is not needed (for example in using medical data to provide an auditory diagnosis); a measure on how much the data is interpreted correctly, even in a binary context, seems important to assess the communicational goal of auditory data. However, this might not be the case in other datasets and audio explorations, where a rise in the user's involvement and participation, either in the mapping exploration or as a part of the design/mapping process, could provide new perspectives and growth opportunities for the scientific field. With this perspective, we can say that the Audio Mostly community has a slight broader range of user-focused methods, but not significant to draw a major distinction between communities.

\section{SYNTHESIS OF AN EVALUATION FRAMEWORK}

The moderate distinctions between both communities showed a major difference between the evaluation structure, which can be user-centred or system-based. The wide spectrum of user exploration and involvement in the evaluation process can be explored in multiple dimensions, in terms of role, range of actions and type of exploration, which becomes fundamental to gain insights on multiple dimensions of auditory artefacts.

To elaborate and characterize the dimensions at stake when evaluating auditory artefacts, we synthesized a framework (Table 2), organized according to the main user's action in the process, the transformative capacity of the auditory outputs, the exploration scope and examples of applications in gathered papers. The framework is composed by six main approaches, arranged in six vertical columns and comprised of six horizontal dimensions.

For the horizontal dimensions, the framework is divided in six analytical dimensions:

(1) "Sequence": the first dimension differentiates the mapping capability to react and mould a given parameter to the user's actions. It may vary from a fixed to a variable sequence depending on the number of outputs.

(2) "Outputs/pathways": this dichotomy compares the number of possible outputs (directly related to the sequence type), with the number of exploration paths in the space of possibilities. If the sequence is fixed, the exploration can also be fixed, or diverge into several pathways to reach the expected result. Several outputs allow several pathways of exploration, and even allow the user to transform the initial outputs and change the space of possibilities.

(3) "Symbolic representation": a visual representation of the second dimension, through abstract forms of possible exploration paths;

(4) "User's role": the fourth dimension distinguishes the user's attitude as a passive, uninvolved listener, or an active, participating agent who holds control of the experience;

(5) "Intention-action": this axis represents how the evaluation begins with the user's intention to interpret the auditory artefact (left), and is progressively replaced by an action-driven experience of the artefact (right), evolving from intention to action in the production of meaning;
(6) "Examples": the last row lists a series of studies found in the literature that are representative of each evaluation approach.

These six dimensions characterize the six main approaches, according to the user's action and involvement:

(1) "LISTENING": the first column describes situations where the user actively listens with no direct involvement, exposed to the artefact as a main spectator in an auditory journey with a linear flow and a single ending;

(2) "ACTIVE LISTENING": the second column describes variations where the user begins to gain an intention for interpreting the auditory stimulus; in this case, a mild exploration already exists that nurtures this attitude, primarily in the temporal dimension of the artefact;

(3) "SEARCH LISTENING": the third column describes scenarios where sound is the conductor of the experience, offering variable outputs depending on the user's exploration paths and how he/she searches into the space of predefined possibilities;

(4) "LISTENING while doing": the fourth column evolves from the previous one in a scenario where the user's actions transform the space of exploration possibilities, with the mapping process adapting and evolving with the user's choices/movements;

(5) "DOING while listening": the fifth column describes nonoriented, action-driven scenarios as conductors of the experience, in which auditory responses lead the exploration and reinforce perception;

(6) "DOING": the sixth column considers listening as a secondary action in the experience, with the user becoming a passive listener in a scenario where sound has a secondary and expendable role.

\section{DISCUSSION}

The proposed framework is primarily divided into six approaches associated with the user's interaction with the auditory artefact.

The first approach, named listening, moulds the user as a mere spectator of the auditory output. In these cases, there is a single auditory sequence as a unique, fixed output, with no possible changes besides the designer's choice in the mapping, either in the sequence of events or the mapping parameters. Around $65 \%$ of the revised ICAD papers fit this approach, including two examples listed in the framework [22, 57] and one from Audio Mostly [10], which resort to listening tests with goal-oriented tasks to test the system performance and mapping success. The first example [57] performed a laboratory study with 6 participants on how could they differentiate different oxygen saturation levels through a sonification. The second [22] was a comparison between two data-to-sound mappings through ranking audio-cued security levels of WiFi networks. The third [10] was an online listening test to evaluate the performance of models of similarity for drum kit patterns.

The second approach, named active listening, grows from the first in terms of exploration possibilities of the fixed sequence, primarily its temporal dimension. For example, actions as rewind, reverse or change the tempo of a given piece do not change the auditory structure listened, but allows a flexibility in the navigation 
Table 2: Proposed framework of evaluation approaches

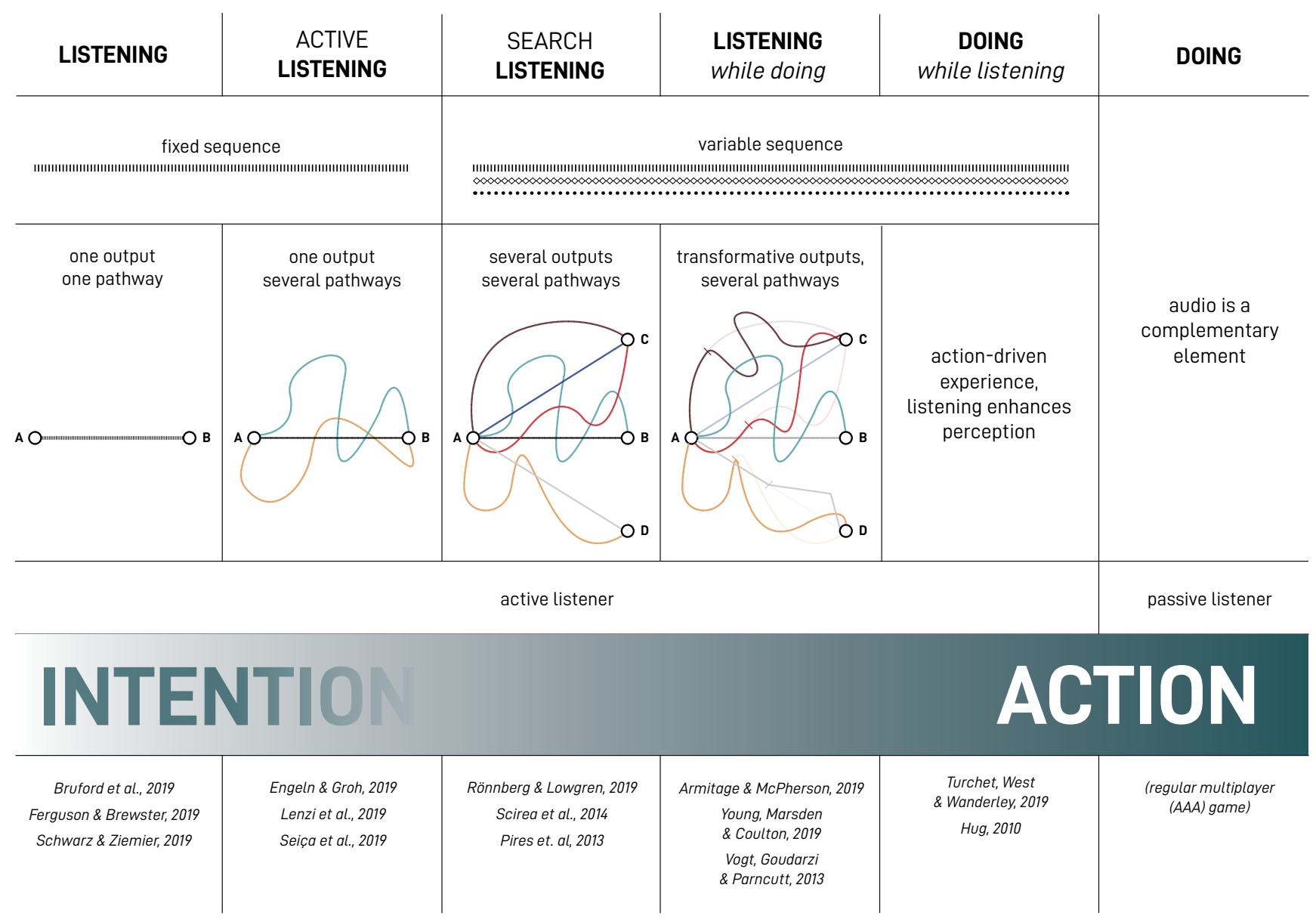

and a beginning active attitude from the listener to explore the composition in a linear way. Several examples of user studies are already encompassed in this approach $[21,38,60]$, with their growing complexity expanding them to the following approaches. The first example [60] devised an exploratory timeline of a sonification, interviewing each user during the exploration using a phenomenological approach. The second example [21] devised a study where users freely explored a physical space to test the effects of acoustic metamaterials. The last example [38] tested a sonification-based system incorporating its use in the users' daily routine, applying questionnaires and semi-structured interviews to collect qualitative analysis and user feedback.

The third approach, named search listening, demands a significant active attitude from the user, with the sequence growing from fixed to variable, which produces a multitude of outputs depending on the user's exploration. Freedom is then given to explore the predefined space of possibilities, with the user's intention for interpretation starting to become action for interpretation for producing meaning. Interactive installations are an example, as Photone, which combines colored images with musical sonification [55] as well as scene-focused playtesting, exploring the relationship of procedurally generated music with the player's experience of narrative [58], or an experience of an audio-based soundscape game to elicit sensoriality and sensemaking [49].

The fourth approach, named listening while doing, has a strong focus on action for the meaning-making process, with the user producing changes in the composition as a consequence. The main difference between the third and fourth approaches is that the user does not just changes the output: he/she can change the designer's mapping choices, transforming the acoustic relations devised to represent the data and becoming the designer of his/her own experience. Methods of participatory design enter this spectrum of action, where the designers do not exactly know beforehand the results they will achieve, and enter a co-creative environment for acquiring knowledge. The works of $[3,67,75]$ are examples of this approach, with participatory workshops for redesigning a partlyfinished digital musical instrument [3], for designing an explorative installation using the "emotive qualities of sound to communicate the visceral experience of womanhood"[75], and for co-creative 
sonification design to evaluate different quantitative evaluation criteria [67].

The fifth column, named doing while listening, refers to scenarios where the listening process becomes a secondary process, dominated by the action that defines the experience. Although the focus shifts entirely from listening to action, the auditory dimension is still a key element for perception and for enhancing the action taken. Performance events to evaluate new prototypes for musical expression fit this approach, as the first example used to evaluate a smart mandolin performer and respective audience with haptic gilets [64], and the second to explore performative soundmaking on the design process of prototypes [31].

The sixth and last column, named doing, focuses on action-driven experience which may or may not have an auditory dimension. The sound element, although evident, is complementary and can be detached from the main experience without prejudices. Multiplayer, triple A games, where many players compete in professional tournaments as a team, end up turning off the music, as it becomes boring, repetitive and distractive from the communication between teammates and the leader.

The intention-action axis guides the structure of the framework, growing from the user's intention to interpret what he/she hears, which gradually evolves to action-driven impetus to uncover meanings and become an active participant in the design process. This perspective is connected to the expansion of the interpretation spectrum. The focus can be designer's interpretation, changing the artefact so that the user's view can match the designer's; the user's interpretation, changing the artefact to "better support user's preferred interpretation" [61]; or a combination of both so that the artefact is flexible and can "incorporate and balance multiple, perhaps conflicting interpretations and processes of interpretation in design and evaluation" [61].

\section{CONCLUSION AND FUTURE DIRECTIONS}

The current framework we propose focuses on the classification of evaluation approaches according to the main action the user is intended to perform to explore the auditory artefact. By shifting the focus to the user's action, it enhances the purpose of the research field for "facilitating communication or interpretation" [35] to gradually incorporate the user's experience of the acoustic data.

This proposal was a first iteration of the conducted literature review and reflection upon the evaluation tendencies when comparing both communities. For future work, we intend to expand the assessment, reviewing more thoroughly the selected conferences, and include other sound-related, interaction-based and artistic-based conferences, as well as highly regarded journals. For the framework, we seek to review each study and the resulting outputs to establish a correlation between the expected results and intentions of each proposed approach, and the results each one can provide.

We expect this framework fosters new perspectives and expands the scope of research of auditory displays, reinforcing the consolidation and solid acknowledgment of the field.

\section{ACKNOWLEDGMENTS}

This work is funded by national funds through the FCT - Foundation for Science and Technology, I.P., within the scope of the project CISUC - UID/CEC/00326/2020 and by European Social Fund, through the Regional Operational Program Centro 2020. The first author is also funded by the FCT - Foundation for Science and Technology, under the grant SFRH/BD/138285/2018.

\section{REFERENCES}

[1] Robert L Alexander, Sile O'Modhrain, Jason A Gilbert, and Thomas H Zurbuchen. 2014. Auditory and Visual Evaluation of Fixed-Frequency Events in Time-Varying Signals. In In Prooceedings of the 20th International Conference on Auditory Display (ICAD 2014). Georgia Institute of Technology.

[2] Areti Andreopoulou and Brian FG Katz. 2015. On the use of subjective htrf evaluations for creating global perceptual similarity metrics of assessors and assessees. In In Prooceedings of the 21st International Conference on Auditory Display (ICAD 2015). Georgia Institute of Technology.

[3] Jack Armitage and Andrew McPherson. 2019. Bricolage in a Hybrid Digital Lutherie Context: A Workshop Study. In Proceedings of the 14th International Audio Mostly Conference: A fourney in Sound (Nottingham, United Kingdom) (AM'19). Association for Computing Machinery, New York, NY, USA, 82-89. https://doi.org/10.1145/3356590.3356604

[4] Eliott Audry and Jérémie Garcia. 2019. Congruent audio-visual alarms for supervision tasks. In In Prooceedings of the 25th International Conference on Auditory Display (ICAD 2019). Georgia Institute of Technology.

[5] Juan Pablo Martinez Avila, Adrian Hazzard, Chris Greenhalgh, and Steve Benford. 2019. Augmenting Guitars for Performance Preparation. In Proceedings of the 14th International Audio Mostly Conference: A fourney in Sound (Nottingham, United Kingdom) (AM'19). Association for Computing Machinery, New York, NY, USA, 69-75. https://doi.org/10.1145/3356590.3356602

[6] Nida Aziz, Tony Stockman, and Rebecca Stewart. 2019. An investigation into customisable automatically generated auditory route overviews for pre-navigation. In In Prooceedings of the 25th International Conference on Auditory Display (ICAD 2019). Georgia Institute of Technology.

[7] Brandon Biggs, James M Coughlan, and Peter Coppin. 2019. Design and evaluation of an audio game-inspired auditory map interface. In In Prooceedings of the 25th International Conference on Auditory Display (ICAD 2019). Georgia Institute of Technology.

[8] Derek Brock, S Camille Peres, and Brian McClimens. 2012. Evaluating listeners' attention to and comprehension of serialy interleaved, rate-accelerated speech. In In Prooceedings of the 18th International Conference on Auditory Display (ICAD 2012). Georgia Institute of Technology.

[9] Gary Bromham, David Moffat, Mathieu Barthet, Anne Danielsen, and György Fazekas. 2019. The Impact of Audio Effects Processing on the Perception of Brightness and Warmth. In Proceedings of the 14th International Audio Mostly Conference: A fourney in Sound (Nottingham, United Kingdom) (AM'19). Association for Computing Machinery, New York, NY, USA, 183-190. https: //doi.org/10.1145/3356590.3356618

[10] Fred Bruford, Mathieu Barthet, SKoT McDonald, and Mark Sandler. 2019. Modelling Musical Similarity for Drum Patterns: A Perceptual Evaluation. In Proceedings of the 14th International Audio Mostly Conference: A fourney in Sound (Nottingham, United Kingdom) (AM'19). Association for Computing Machinery, New York, NY, USA, 131-138. https://doi.org/10.1145/3356590.3356611

[11] Ivica Ico Bukvic, Gregory Earle, Disha Sardana, and Woohun Joo. 2019. Studies in spatial aural perception: establishing foundations for immersive sonification. In In Prooceedings of the 25th International Conference on Auditory Display (ICAD 2019). Georgia Institute of Technology.

[12] Linda Candy, Shigeki Amitani, and Zafer Bilda. 2006. Practice-led strategies for interactive art research. CoDesign: International fournal of CoCreation in Design and the Arts 2, 4 (2006), 209-223.

[13] Filippo Carnovalini and Antonio Rodà. 2019. A Real-Time Tempo and Meter Tracking System for Rhythmic Improvisation. In Proceedings of the 14th International Audio Mostly Conference: A fourney in Sound (Nottingham, United Kingdom) (AM'19). Association for Computing Machinery, New York, NY, USA, 24-31. https://doi.org/10.1145/3356590.3356596

[14] Juliet M Corbin and Anselm Strauss. 1990. Grounded theory research: Procedures, canons, and evaluative criteria. Qualitative sociology 13, 1 (1990), 3-21.

[15] Stuart Cunningham, Harrison Ridley, Jonathan Weinel, and Richard Picking. 2019. Audio Emotion Recognition Using Machine Learning to Support Sound Design. In Proceedings of the 14th International Audio Mostly Conference: A fourney in Sound (Nottingham, United Kingdom) (AM'19). Association for Computing Machinery, New York, NY, USA, 116-123. https://doi.org/10.1145/3356590.3356609

[16] Stuart Cunningham, Jonathan Weinel, Shaun Roberts, Vic Grout, and Darryl Griffiths. 2013. Initial Objective Subjective Evaluation of a Similarity-Based Audio Compression Technique. In Proceedings of the 8th Audio Mostly Conference (Piteå, Sweden) (AM '13). Association for Computing Machinery, New York, NY, USA, Article 1, 6 pages. https://doi.org/10.1145/2544114.2544116 
[17] Palle Dahlstedt and Ami Skånberg Dahlstedt. 2019. OtoKin: Mapping for Sound Space Exploration through Dance Improvisation. In International Conference on New Interfaces for Musical Expression - NIME2019. Porto Alegre, Brazil.

[18] Alejandro Delgado, SKoT McDonald, Ning Xu, and Mark Sandler. 2019. A New Dataset for Amateur Vocal Percussion Analysis. In Proceedings of the 14th International Audio Mostly Conference: A fourney in Sound (Nottingham, United Kingdom) (AM'19). Association for Computing Machinery, New York, NY, USA 17-23. https://doi.org/10.1145/3356590.3356844

[19] David Dewhurst. 2019. The design and exploration of auditory display effects for blind drivers in autonomous vehicles. In In Prooceedings of the 25th International Conference on Auditory Display (ICAD 2019). Georgia Institute of Technology.

[20] Milena Droumeva and Iain McGregor. 2014. A Method for Comparative Evaluation of Listening to Auditory Displays by Designers and Users. In In Prooceedings of the 20th International Conference on Auditory Display (ICAD 2014). Georgia Institute of Technology.

[21] Lars Engeln and Rainer Groh. 2019. CoHEARence: A Qualitive User-(Pre-)Test on Resynthesized Shapes for Coherent Visual Sound Design. In Proceedings of the 14th International Audio Mostly Conference: A fourney in Sound (Nottingham, United Kingdom) (AM'19). Association for Computing Machinery, New York, NY, USA, 98-102. https://doi.org/10.1145/3356590.3356606

[22] Jamie Ferguson and Stephen Brewster. 2019. Evaluating the magnitude estimation approach for designing sonification mapping topologies. In In Prooceedings of the 25th International Conference on Auditory Display (ICAD 2019). Georgia Institute of Technology.

[23] Thomas J. Graham, Thor Magnusson, Chinmay Rajguru, Arash Pour Yazdan, Alex Jacobs, and Gianluca Memoli. 2019. Composing Spatial Soundscapes Using Acoustic Metasurfaces. In Proceedings of the 14th International Audio Mostly Conference: A fourney in Sound (Nottingham, United Kingdom) (AM'19). Association for Computing Machinery, New York, NY, USA, 103-110. https: //doi.org/10.1145/3356590.3356607

[24] Annaliese Micallef Grimaud, Tuomas Eerola, and Nick Collins. 2019. EmoteControl: A System for Live-Manipulation of Emotional Cues in Music. In Proceedings of the 14th International Audio Mostly Conference: A fourney in Sound (Nottingham, United Kingdom) (AM'19). Association for Computing Machinery, New York, NY, USA, 111-115. https://doi.org/10.1145/3356590.3356608

[25] Brian Hansen, Leya Breanna Baltaxe-Admony, Sri Kurniawan, and Angus G Forbes. 2019. Exploring sonic parameter mapping for network data structures. In In Prooceedings of the 25th International Conference on Auditory Display (ICAD 2019). Georgia Institute of Technology.

[26] Yliess Hati, Francis Rousseaux, and Clément Duhart. 2019. Text-driven mouth animation for human computer interaction with personal assistant. In In Prooceedings of the 25th International Conference on Auditory Display (ICAD 2019). Georgia Institute of Technology.

[27] Laurie M Heller, Arley Schenker, Pulkit Grover, Madeline Gardner, and Felix Liu. 2017. Evaluating Two Ways to Train Sensitivity to Echoes to Improve Echolocation. In In Prooceedings of the 23rd International Conference on Auditory Display (ICAD 2017). Georgia Institute of Technology.

[28] Thomas Hermann. 2008. Taxonomy and definitions for sonification and auditory display. In Proceedings of the 14th International Conference on Auditory Display.

[29] Thomas Hermann, Andy Hunt, and John Neuhoff. 2011. The Sonification Handbook. Logos Verlag, Berlin, Germany.

[30] Thomas Hermann and Marian Weger. 2019. Data-driven Auditory Contrast Enhancement for everyday sounds and sonifications. In In Prooceedings of the 25th International Conference on Auditory Display (ICAD 2019). Georgia Institute of Technology.

[31] Daniel Hug. 2010. Performativity in Design and Evaluation of Sounding In teractive Commodities. In Proceedings of the 5th Audio Mostly Conference: A Conference on Interaction with Sound (Piteå, Sweden) (AM '10). Association for Computing Machinery, New York, NY, USA, Article 7, 8 pages. https: //doi.org/10.1145/1859799.1859806

[32] D. Idhe. 1977. Experimental Phenomenology. State University of New York Press, New York, USA, Chapter Phenomena and the Phenomenological Reductions, 29-54.

[33] Rébecca Kleinberger, Stefanakis George, and Sebastian Franjou. 2019. Speech companions: Evaluating the effects of musically modulated auditory feedback on the voice. In In Prooceedings of the 25th International Conference on Auditory Display (ICAD 2019). Georgia Institute of Technology.

[34] Takanori Komatsu and Eiji Hayashi. 2019. A design guide-line of auditory display for electric appliance. In In Prooceedings of the 25th International Conference on Auditory Display (ICAD 2019). Georgia Institute of Technology.

[35] Gregory Kramer, BN Walker, Terri Bonebright, Perry Cook, J Flowers, Nadine Miner, John Neuhoff, R Bargar, S Barrass, J Berger, et al. 1999. The sonification report: Status of the field and research agenda. report prepared for the national science foundation by members of the international community for auditory display. International Community for Auditory Display (ICAD), Santa Fe, NM (1999).

[36] Thimmaiah Kuppanda, Norberto Degara, David Worrall, Balaji Thoshkahna, and Meinard Müller. 2015. Virtual reality platform for sonification evaluation. In In
Prooceedings of the 21st International Conference on Auditory Display (ICAD 2015). Georgia Institute of Technology.

[37] Adam Lefaivre and John Z. Zhang. 2018. Music Genre Classification: GenreSpecific Characterization and Pairwise Evaluation. In Proceedings of the Audio Mostly 2018 on Sound in Immersion and Emotion (Wrexham, United Kingdom) (AM'18). Association for Computing Machinery, New York, NY, USA, Article 22, 4 pages. https://doi.org/10.1145/3243274.3243310

[38] Sara Lenzi, Ginevra Terenghi, Riccardo Taormina, Stefano Galelli, and Paolo Ciuccarelli. 2019. Disclosing cyber attacks on water distribution systems: an experimental approach to the sonification of threats and anomalous data. In In Prooceedings of the 25th International Conference on Auditory Display (ICAD 2019). Georgia Institute of Technology.

[39] Stan Lester. 1999. An introduction to phenomenological research.

[40] Grace Li and Bruce N Walker. 2019. Mixed speech and non-speech auditory displays: impacts of design, learning, and individual differences in musical engagement. In In Prooceedings of the 25th International Conference on Auditory Display (ICAD 2019). Georgia Institute of Technology.

[41] Peter Lundén, Marja Gustin, Mats E. Nilsson, Jens Forssén, and Björn Hellström. 2010. Psychoacoustic Evaluation as a Tool for Optimization in the Development of an Urban Soundscape Simulator. In Proceedings of the 5th Audio Mostly Conference: A Conference on Interaction with Sound (Piteå, Sweden) (AM '10). Association for Computing Machinery, New York, NY, USA, Article 3, 6 pages. https://doi.org/ $10.1145 / 1859799.1859802$

[42] Keenan R May, Briana Sobel, Jeff Wilson, and Bruce N Walker. [n.d.]. Auditory displays to facilitate object targeting in 3D space. In In Prooceedings of the 25th International Conference on Auditory Display (ICAD 2019). Georgia Institute of Technology.

[43] Iain McGregor, Gregory Leplâtre, Phil Turner, and Tom Flint. 2010. Soundscape Mapping: a tool for evaluating sounds and auditory environments. (2010).

[44] Neil McGuiness and Chris Nash. 2019. The Pulse: Embedded Beat Sensing Using Physical Data. In Proceedings of the 14th International Audio Mostly Conference: A fourney in Sound (Nottingham, United Kingdom) (AM'19). Association for Computing Machinery, New York, NY, USA, 207-214. https://doi.org/10.1145/ 3356590.3356621

[45] Yuki Nakayama, Yuji Takano, Masaki Matsubara, Kenji Suzuki, and Hiroko Terasawa. 2015. Real-time smile sonification using surface EMG signal and the evaluation of its usability. In In Prooceedings of the 21st International Conference on Auditory Display (ICAD 2015). Georgia Institute of Technology.

[46] Timothy Neate, Norberto Degara, Andy Hunt, and Frederik Nagel. 2014. A Generic Evaluation Model for Auditory Feedback in Complex Visual Searches. In In Prooceedings of the 20th International Conference on Auditory Display (ICAD 2014). Georgia Institute of Technology.

[47] John G Neuhoff. 2019. Is sonification doomed to fail?. In In Prooceedings of the 25th International Conference on Auditory Display (ICAD 2019). Georgia Institute of Technology.

[48] Richard Pinsenschaum and Flaithri Neff. 2016. Evaluating Gesture Characteristics When Using a Bluetooth Handheld Music Controller. In Proceedings of the Audio Mostly 2016 (Norrköping, Sweden) (AM '16). Association for Computing Machinery, New York, NY, USA, 209-214. https://doi.org/10.1145/2986416.2986443

[49] Durval Pires, Bárbara Furtado, Tiago Carregã, Luís Reis, Luís Lucas Pereira, Rui Craveirinha, and Licínio Roque. 2013. The blindfold soundscape game: a case for participation-centered gameplay experience design and evaluation. In Proceedings of the 8th Audio Mostly Conference. 1-7.

[50] Zuzanna Podwinska, Bruno M Fazenda, and William J Davies. 2019. Testing spatial aspects of auditory salience. In In Prooceedings of the 25th International Conference on Auditory Display (ICAD 2019). Georgia Institute of Technology.

[51] Cornelius Poepel. 2014. Evaluating Computer-Based Musical Instruments from the Perspective of Listening. In Proceedings of the 9th Audio Mostly: A Conference on Interaction With Sound (Aalborg, Denmark) (AM '14). Association for Computing Machinery, New York, NY, USA, Article 19, 8 pages. https: //doi.org/10.1145/2636879.2636900

[52] Winters R Michael, Ankur Kalra, and Bruce N Walker. [n.d.]. Hearing artificial intelligence: Sonification guidelines \& results from a case-study in melanoma diagnosis. In In Prooceedings of the 25th International Conference on Auditory Display (ICAD 2019). Georgia Institute of Technology.

[53] Richard Ramchurn, Juan Martinez-Avila, Sarah Martindale, Alan Chamberlain, Max L Wilson, and Steve Benford. 2019. Improvising a Live Score to an Interactive Brain-Controlled Film. In Proceedings of the International Conference on New Interfaces for Musical Expression. Porto Alegre, Brazil: Federal University of Rio Grande do Sul.

[54] Katja Rogers and Michael Weber. 2019. Audio Habits and Motivations in Video Game Players. In Proceedings of the 14th International Audio Mostly Conference: A fourney in Sound (Nottingham, United Kingdom) (AM'19). Association for Computing Machinery, New York, NY, USA, 45-52. https://doi.org/10.1145/ 3356590.3356599

[55] Niklas Rönnberg and Jonas Lowgren. 2019. Traces of modal synergy: studying interactive musical sonification of images in general-audience use. In In Prooceedings of the 25th International Conference on Auditory Display (ICAD 2019). Georgia 
Institute of Technology.

[56] Richard Savery, Madhukesh Ayyagari, Keenan May, and Bruce N Walker. 2019 Soccer sonification: Enhancing viewer experience. In In Prooceedings of the 25th International Conference on Auditory Display (ICAD 2019). Georgia Institute of Technology.

[57] Sebastian Schwarz and Tim Ziemer. 2019. A psychoacoustic sound design for pulse oximetry. In In Prooceedings of the 25th International Conference on Auditory Display (ICAD 2019). Georgia Institute of Technology.

[58] Marco Scirea, Yun-Gyung Cheong, Mark J. Nelson, and Byung-Chull Bae. 2014. Evaluating Musical Foreshadowing of Videogame Narrative Experiences. In Proceedings of the 9th Audio Mostly: A Conference on Interaction With Sound (Aalborg, Denmark) (AM '14). Association for Computing Machinery, New York, NY, USA, Article 8, 7 pages. https://doi.org/10.1145/2636879.2636889

[59] Gabriela Seibert and Daniel Hug. 2013. Bringing Musicality to Movement Sonification: Design and Evaluation of an Auditory Swimming Coach. In Proceedings of the 8th Audio Mostly Conference (Piteå, Sweden) (AM '13). Association for Computing Machinery, New York, NY, USA, Article 17, 6 pages. https://doi.org/10.1145/2544114.2544127

[60] Mariana Seiça, Pedro Martins, Licínio Roque, and F Amílcar Cardoso. 2019. A sonification experience to portray the sounds of portuguese consumption habits. In In Prooceedings of the 25th International Conference on Auditory Display (ICAD 2019). Georgia Institute of Technology.

[61] Phoebe Sengers and Bill Gaver. 2006. Staying open to interpretation: engaging multiple meanings in design and evaluation. In Proceedings of the 6th conference on Designing Interactive systems. 99-108.

[62] Tony Stockman and Dena Al-Thanki. 2010. Development and Evaluation of Cross-Modal XML Schema Browser. In In Prooceedings of the 16th International Conference on Auditory Display (ICAD 2010). Georgia Institute of Technology.

[63] Elio Toppano, Sveva Toppano, and Alessandro Basiaco. 2019. Moving across Sonic Atmospheres. In Proceedings of the 14th International Audio Mostly Conference: A Journey in Sound (Nottingham, United Kingdom) (AM'19). Association for Computing Machinery, New York, NY, USA, 139-146. https://doi.org/10.1145/ 3356590.3356612

[64] Luca Turchet, Travis West, and Marcelo M. Wanderley. 2019. Smart Mandolin and Musical Haptic Gilet: Effects of Vibro-Tactile Stimuli during Live Music Performance. In Proceedings of the 14th International Audio Mostly Conference: A fourney in Sound (Nottingham, United Kingdom) (AM'19). Association for Computing Machinery, New York, NY, USA, 168-175. https://doi.org/10.1145/ 3356590.3356616

[65] P. Vickers and B. Hogg. 2006. Sonification Abstraite / Sonification Concr'ete: An "Aesthetic Perspective Space" for Classifying Auditory Displays in the Ars Musica Domain. In Proceedings of the 12th International Conference on Auditory Display. London, UK, 210-216.

[66] Paul Vickers and Robert Höldrich. 2019. Direct Segmented Sonification of Char acteristic Features of the Data Domain. In In Prooceedings of the 25th International Conference on Auditory Display (ICAD 2019). Georgia Institute of Technology.

[67] K. Vogt. 2014. A Quantitative Evaluation Approach to Sonifications. In Proceedings of the International Conference on Auditory Display (ICAD 2014). Budapest, Hungary.

[68] K. Vogt, V. Goudarzi, and R. Parncutt. 2013. Empirical Aesthetic Evaluation of Sonifications. In Proceedings of the International Conference on Auditory Display (ICAD 2013). Lodz, Poland, 175-179.

[69] Bartlomiej P Walus, Sandra Pauletto, and Amanda Mason-Jones. 2015. Evaluating the use of sonification and music to support the communication of alcohol health risk to young people: initial results. In In Prooceedings of the 21st International Conference on Auditory Display (ICAD 2015). Georgia Institute of Technology.

[70] Marian Weger, Thomas Hermann, and Robert Höldrich. 2019. Real-time auditory contrast enhancement. In In Prooceedings of the 25th International Conference on Auditory Display (ICAD 2019). Georgia Institute of Technology.

[71] Marian Weger and Robert Höldrich. 2019. A Hear-through System for Plausible Auditory Contrast Enhancement. In Proceedings of the 14th International Audio Mostly Conference: A fourney in Sound (Nottingham, United Kingdom) (AM'19). Association for Computing Machinery, New York, NY, USA, 1-8. https://doi. org $/ 10.1145 / 3356590.3356593$

[72] György Wersényi, Hunor Nagy, and Ádám Csapó. 2015. Evaluation of reaction times to sound stimuli on mobile devices. In In Prooceedings of the 21st International Conference on Auditory Display (ICAD 2015). Georgia Institute of Technology.

[73] Dirk Vander Wilt and Morwaread Mary Farbood. 2019. Automating Audio Description for Live Theater: Using Reference Recordings to Trigger Descriptive Tracks in Real Time. In Proceedings of the 14th International Audio Mostly Conference: A fourney in Sound (Nottingham, United Kingdom) (AM'19). Association for Computing Machinery, New York, NY, USA, 75-81. https://doi.org/10.1145/ 3356590.3356603

[74] KatieAnna Wolf and Rebecca Fiebrink. 2019. Toward Supporting End-User Design of Soundscape Sonifications. In In Prooceedings of the 25th International Conference on Auditory Display (ICAD 2019). Georgia Institute of Technology.
[75] Emma Young, Alan Marsden, and Paul Coulton. 2019. Making the Invisible Audible: Sonifying Qualitative Data. In Proceedings of the 14th International Audio Mostly Conference: A fourney in Sound. 124-130. 